\title{
Entre deux patries
}

\section{Lidia Mihova}

\section{OpenEdition \\ Journals}

Édition électronique

URL : https://journals.openedition.org/cher/10413

DOI : $10.4000 /$ cher. 10413

ISSN : 2803-5992

\section{Éditeur}

Presses universitaires de Strasbourg

\section{Édition imprimée}

Date de publication : 1 décembre 2013

Pagination : 203-209

ISBN : 978-2-86820-560-5

ISSN : 1968-035X

Référence électronique

Lidia Mihova, «Entre deux patries », reCHERches [En ligne], 11 | 2013, mis en ligne le 08 février 2022, consulté le 09 février 2022. URL : http://journals.openedition.org/cher/10413 ; DOI : https://doi.org/ 10.4000/cher.10413

\section{(ब)(}

Ce(tte) œuvre est mise à disposition selon les termes de la Licence Creative Commons Attribution Pas d'Utilisation Commerciale - Partage dans les Mêmes Conditions 4.0 International. 


\title{
Entre deux patries
}

\author{
LIDIA MIHOVA \\ Université de Strasbourg
}

\begin{abstract}
Tombreux sont les points de la carte européenne où découvrir une 1 population bulgare plus ou moins dense à l'époque de la domination ottomane sur les Balkans. On connaît quelques-unes des vagues massives de population bulgare dans l'Empire austro-hongrois, en Roumanie ou sur le territoire de la Russie. Une partie non négligeable des Bulgares émigrés sous le joug ottoman rentrent au pays après la Libération en 1878 mais un bon nombre restent entre eux sur les terres où ils avaient trouvé refuge et qu'ils considéraient comme leur "chez soi». Le sentiment d'appartenance à une ethnie et à la "vieille» patrie ne quittera point certains d'entre eux durant toutes ces années et se transmettra même de génération en génération comme c'est le cas pour les Bulgares de Bessarabie par exemple.

En Moldavie, dans la région Taraclie se trouve le village Valea Perjei peuplé du début du XIX ${ }^{\text {e }}$ siècle à nos jours de Moldaves et de Bulgares. Selon une légende locale du début du XIX ${ }^{e}$ siècle, des Moldaves sont venus s'installer sur ces terres pour s'affranchir de leurs féodaux (Malechkova, Dimov, Kourtev, $1998: 8)$. En ce lieu où se trouvait déjà un village tartare, ils se sont sentis en sécurité, ils ont trouvé une terre fertile et des sources naturelles. Ils ont donné au village le nom de Valea Perjei car il y avait là des pruniers. Plus tard, en 1812, les premiers Bulgares sont venus dans ce village: ils étaient sans doute originaires de villages autour de Sliven et Yambol en Bulgarie. Moldaves et Bulgares ont commencé à cohabiter, partageant petits tracas et vie de tous les jours et les guerres, les révolutions, les années de pénurie et de famine, les changements en bien comme en mal. Tous parlaient le bulgare, le moldave et le russe qui fut longtemps la langue officielle de la population dans la région.
\end{abstract}


Au fil du temps Moldaves et Bulgares se sont mariés, ont célébré ensemble leurs fêtes respectives présentant souvent, aux yeux des ethnologues des points communs à ces deux cultures traditionnelles. Récemment, la crise a poussé beaucoup d'habitants de Valea Perjei à quitter leurs terres natales pour chercher du travail à l'étranger: le village compte aujourd'hui 6000 habitants dont un tiers sont des Bulgares. Durant ces années, ces derniers sont parvenus à sauvegarder leur identité bulgare en même temps que leur langue. Ils ont construit une église et peu après une école où l'on a enseigné le bulgare. On suppose que cette école existait déjà en 1831, date à laquelle les Bulgares ont obtenu la citoyenneté russe (Malechkova, Dimov, Kourtev, 1998: 37). La première rencontre des descendants de ces Bulgares avec des représentants de «la patrie des ancêtres» comme les gens du lieu nomment la Bulgarie a eu lieu en 1967 quand un groupe de Bulgares de Stara Zagora est arrivé à Valea Perjei. "C'a été un miracle pour les habitants de Valea Perjei» disent les chroniqueurs de l'histoire du village quand ils décrivent les émouvantes retrouvailles entre compatriotes. (Malechkova, Dimov, Kourtev, 1998: 37). C'est à la suite de cet événement que notamment les relations avec la Bulgarie et la Roumanie sont devenues fréquentes jusqu'à se poursuivre parfois dans les universités bulgares et roumaines.

Anna Malechkova est une Bulgare de Bessarabie née en 1943 à Valea Perjei en Moldavie. Après avoir fait ses études à l'Institut pédagogique de Tiraspol Tarass Chevtchenko, elle travaille dans les rédactions de divers périodiques et revues en Moldavie. Elle se marie à un Bulgare de Bulgarie et depuis 1971, elle habite la Bulgarie. Son frère Nicolas Kourtev né en 1947 toujours à Valea Perjei, a fait ses études en philologie russe à l'Institut pédagogique Taras G. Chevetchenko. Il a travaillé comme professeur, il a été élu pour deux mandats non consécutifs maire du village Valeia Perjei. Ensuite il a soutenu sa thèse de doctorat à l'Institut de langue bulgare auprès de l'Academie bulgare des Sciences. Il a été recteur-adjoint à l'université d'État à Taracli et aujourd'hui il est enseignant habilité à l'université en continuant ses recherches linguistiques, anthropologiques et folkloriques sur les Bulgares de Bessarabie.

Anna Malechkova et son frère Nicolas Kourtev ont publié un calendrier historique intitulé Ce jour-là en Bessarabie: ils ont aussi écrit et fait paraître les romans: Trouver la formule magique (Yambol, 1998) et Le rire du diable (Sliven, 2000). En collaboration avec Dimitar Dimov, ils ont fait connaître l'histoire de leur village natal: Valea Perjei (1998). Anna Malechkova est aussi l'auteure de recueils de poèmes et de livres pour enfants. 
Avant son arrivée en Bulgarie, dit-elle dans une conversation privée, elle a écrit et publié des récits et poèmes en russe mais une fois installée dans son pays natal, elle a commencé de ne plus écrire qu'en bulgare. Son œuvre, son activité d'éditrice sont étroitement liées à la Bessarabie et à son village natal: Valea Perjei. Actuellement, elle prépare un recueil de proverbes bessarabiens sur lequel elle travaille depuis plus de dix ans déjà, tandis que son frère travail sur un recueil de légendes bessarabiennes.

Sa poésie présente de nombreuses particularités propres à la poésie de Bessarabie sur laquelle se sont déjà penchés des chercheurs. Déjà enregistrée depuis quarante ans, cette poésie est née dans un milieu linguistique étranger, elle a été créée par des gens coupés de l'actualité des processus artistiques et linguistiques affectant le pays - porteur de la langue dans laquelle ils écrivaient. Cette poésie est écrite dans la langue des "ancêtres" transmise de génération en génération pendant deux siècles. Selon Elena Nalbantova, elle a été "créée en absence de sa propre tradition littéraire ${ }^{1}$. Sa configuration est spécifique: thèmes, motifs, langage littéraire. Si la poésie de Anna Malechkova reflète plusieurs particularités propres à la poésie des Bulgares de Bessarabie, elle s'en distingue pourtant. Sans doute en raison du fait qu'elle habite et crée en Bulgarie depuis quarante ans déjà où elle a déjà reçu plusieurs prix pour avoir enrichi la culture bulgare. Pour elle, l'identification a deux espaces «familiers»: la Bulgarie et la Moldavie. En d'autres termes, l'existence à la frontière du "sien» et du "sien», selon la terminologie de Elena Nalbantova, est surchargée et compliquée.

Dans le dernier recueil de poèmes de Anna Malechkova ( $\mathrm{Ce}$ que tu es...», 2011), on retrouve le motif existentiel de l'appartenance parallèle à deux patries, deux espaces culturels dont l'un est «l'autre» que l'on s'approprie et l'autre: le «sien». Cependant, on ne s'y sent pas toujours reconnu ni bien accueilli comme le montrent les poèmes: "Nous sommes bulgares, n'est-ce pas?» ou «Aux Bessarabiens». Le seul titre du recueil fait allusion au chemin douloureux "vers les siens" et sa propre identification à la fois: "Où est mon étoile? / Prédestinée à deux pays / Je la cherche d'une main tremblante...» se confie l'héroïne lyrique. Cette poésie intime et confidentielle a pour qualité principale d'être sincère et vécue. Dans un certain sens, cette lyrique est "narrative» (j'avance cet oxymore) dans la mesure où elle est fidèle au vécu.

1. Nalbantova Elena, http://liternet.bg/publish2/enalbantova/poeziiata.html 
La dernière édition préparée par Anna Malechkova et son frère Nicolas Kourtev est la série littéraire Chroniques bessarabiennes en quatre parties, déjà éditée à Kishinev en bulgare. La série inclut deux de leurs livres précédents auxquels se sont ajoutées deux nouvelles parties. Elle raconte le destin des Bulgares qui, cherchant à échapper au joug ottoman se sont exilés dans les régions périphériques de la Russie tsariste de cette époque. La première partie: Trouver la formule magique décrit la période 1812-1900, les difficultés et épreuves de l'enracinement ainsi que la transformation de terres en friche en jardins fertiles. La deuxième partie: Au carrefour comprend la période 1900-1940, donc l'époque des réformes, la Première Guerre mondiale, la Révolution d'Octobre. On y trouve évoquées aussi la Bessarabie sous occupation du Royaume de Roumanie et la Seconde Guerre mondiale. La deuxième partie: Le rire du diable évoque les années 1940-1953 : époque de répressions, d'internement des émigrés en Sibérie, au Kazakhstan, à Fergana, ville de l'Est de l'Ouzbekistan et en Extrême-Orient. Cette partie évoque le génocide et la famine de 1946-1947, années durant lesquelles ont disparu des familles entières, elle retranscrit la période des répressions systématiques et l'extermination des plus éveillés, parmi les Bulgares. La dernière et quatrième partie intitulée Le Président couvre la période 1953-1993 et elle décrit la formation des cadres socialistes locaux, les espoirs et la destruction des valeurs morales, la décomposition d'une société ainsi que la chute d'un gouvernement négligeant les problèmes des Bulgares et Bessarabiens. Cette série publiée récemment en Moldavie a été accueillie chaleureusement selon Anna Malechkova. Elle a été présentée le 22 mai 2013 à Taraclie à l'occasion de la célébration du bicentenaire de l'exode à Boudjak.

De par leur genre, les quatre volumes réalisent une chronique littéraire historique où se mêlent le documentaire et la fiction, où les faits historiques et personnages réels prennent vie dans un récit alerte riche en émotions. Comme dans l'histoire de Valea Perjei, le refus voulu de se distancer de l'objet de la description - en accord avec la distanciation propre à l'attitude scientifique face à l'histoire - n'enlève rien à la qualité des faits et des documents.

La deuxième partie, déjà connue du public bulgare, est particulièrement intéressante dans son évoquation d'épisodes de la vie en Bessarabie et des événements mal connus. Le sous-titre du livre «Autour de la vie des Bulgares bessarabiens» fait allusion au caractère hybride des genres dans cette œuvre: mélange de documentaire et de fiction. 
Les événements reconstitués ont vraiment eu lieu. Beaucoup de personnages du récit sont présentés avec leurs vrais noms. La peur, le désespoir, la résignation, le mécontentement et l'espoir qui remplissent les pages du livre ne sont guère le fruit d'invention ou de «tour de magie» littéraires, car ils ont réellement existé et ont été vécus par des gens en chair et en os.

Le livre est divisé en deux parties: «La trahison» et «Le rire du diable». La première partie évoque en premier le motif de l'éloignement des racines perçu par les narrateurs comme une trahison. Le conte débute par l'arrivée d'un Bulgare-activiste soviétique ayant participé au prétendu "coup de balai des ennemis du peuple». Il renonce à ses origines bulgares et change de nom en se transformant en ennemi des «siens». Il détruit leur monde, ainsi que tout ce qu'ils avaient conservé et tout ce qui les avait sauvegardés, envoyant en exil les plus entreprenants et intelligents, y compris certains de ceux qui avaient sympathisé avec le pouvoir soviétique.

Ce récit triste et effrayant met en relief les destins de personnages bulgares et moldaves. Par exemple la triste destinée de la famille de Sénia et Stéphane Stoyanov qui, après avoir fait des études à l'étranger (lui aux PaysBas, elle à Genève) rentrent au pays natal avant de se voir séparés de force et exilés avec leurs enfants loin en Sibérie en raison d'on ne sait quelle faute. Ou bien l'histoire d'un Roumain: le pope Oursaki, arrivé au village, avec sa jolie femme, pendant la guerre et l'occupation roumaine, adulé de tous et ne craignant pas d'exprimer des «pensées hérétiques concernant Hitler». "Le pope Oursaki était proche de nous, il n'hésitait pas à entrer dans les maisons des Bessarabiens, à y passer du temps et à bavarder longuement autour d'un verre de vin ». Le pope Oursaki restera au village même après le retrait des occupants roumains, pour partager le sort de ses paroissiens - Bulgares et Moldaves.

La majorité des personnages sont présents dans le récit avec leurs vrais noms: le pope Oursaki et tous ceux qui sont envoyés en exil en Sibérie comme Sénia et ses enfants. Cependant certains noms sont changés, surtout les noms de ceux qui avaient participé aux répressions - nous confie Anna Maléchkova - car leurs proches sont encore en vie.

La deuxième partie de l'œuvre «Le rire du diable» raconte les premières années après la guerre. Elle évoque la famine de 1946-1947, s'efforce de représenter cette tragédie humaine de manière authentique et correcte: les ordres officiels de l'État pour le ramassage du blé, mais aussi le "zèle» excessif des cadres locaux, souvent passé sous silence. 
Chez nous il y avait des gens qui montraient vraiment trop de zèle. Beaucoup trop. On rapportait sans cesse comment les choses se passaient mais le peuple était abandonné

se confie dans une interview Anna Maléchkova et elle ajoute:

Il y a encore des archives que les gens n'osent pas ouvrir car les héritiers de ceux qui commettaient les atrocités les plus terribles sont encore vivants. Et des atrocités il y en avait. ${ }^{2}$

Le blé ramassé grain par grain dont l'absence avait provoqué la mort par la faim de dizaines de gens, est trouvé "oublié» sur le quai d'une gareTchadar-Lunga.

Au printemps 1947 - continue Anna Malechkova - on s'est aperçu avec horreur que ce blé n'avait été envoyé ni en Russie ni où que ce soit, il avait été tout simplement ramassé mais endommagé et on ne pouvait même pas le donner à manger aux animaux. Mauvaise conservation, pas de séchage, rien du tout.

Le livre - dit Anna Malechkova pendant une conversation privée - n'est pas le premier «récit» de la famine vécue en Bessarabie. Déjà dans les années 1960 on avait commencé à aborder le sujet, quoique timidement. Ce sujet, longtemps laissé sous silence par le passé, est désormais sérieusement analysé. Mais, comme dit l'auteure, il s'agit du premier livre en donnant une interprétation littéraire.

Parmi les sources consultées par les auteurs, ne figurent pas les archives de Tchadar-Lunga, Kichinew et Komrat (encore aujourd'hui difficilement accessibles ou en tout cas non accesssibles à tout le monde) mais des documents archivés dans les mairies des villages et surtout les mémoires. Les documents des familles y figurent. Les auteurs les ont recherchés en faisant le tour des villages car ils représentaient pour eux «la mémoire vivante» du peuple.

Les auteurs considèrent leur série comme une lecture littéraire fictionnelle pourtant instructive sur l'histoire des Bulgares de Bessarabie. Ils proposent que leurs textes soient étudiés à l'école, à l'université. Ils sont en effet convaincus - et pour cause - que leur livre vient compléter l'histoire de la Bulgarie.

2. Malechkova, Anna, La mémoire vivante du peuple sur la faim en Bessarabie est la plus affreuse, Interview in Focus, 4 fevrier 2012, http://www.focus-news.net/?id=f20333; Voir aussi : Malechkova, Anna, "Confession», in Koreni Bulgqrski (Des racines bulgares), ${ }^{\circ} 1$, août 2009. 
Les récits historiques des deux Bulgares de Bessarabie interprètent la Grande Histoire (les grands événements historiques) à travers l'évocation $\mathrm{du}$ destin des petites gens ordinaires. Leur narration n'est pas étrangère, au contraire, à leur engagement personnel dans la présentation de faits et événements sous un éclairage d'autodérision, à cause du regard porté sur le vécu, les illusions, les espoirs.

Après les grands bouleversements et la dislocation del'ex-URSS à partir des années $1990 \mathrm{du} \mathrm{XX}^{\mathrm{e}}$ siècle, il est devenu possible de pratiquer des recherches sur l'histoire des communautés de Bulgares expatriés venus s'installer sur le territoire de la Russie tsariste à l'époque de la domination ottomane. Les historiens, ethnologues et linguistes de Bulgarie ont désormais la possibilité de se livrer à des recherches sur le terrain. La littérature des Bulgares de Bessarabie a déjà ses chercheurs comme Svétozar Vassilev et Elena Nalbantova. Ecrire une histoire scientifiquement fondée des Bulgares bessarabiens en considérant celle-ci comme partie intégrante de la grande histoire de la Bulgarie représente un défi à relever pour les scientifiques bulgares. Cette étude historique pourrait être réalisée par un chercheur neutre capable de collecter et de composer la mosaïque des faits, des documents et des récits intimes. Ce scientifique devrait prendre ses distances par rapport à l'objet de sa description et en donner une analyse impartiale.

Alors, l'œuvre de Anna Malechkova et Nicolas Kourtev prendrait la place qu'elle mérite parmi les sources d'information précieuses pour la constitution de l'histoire encore inachevée et incomplète des communautés d'expatriés bulgares. 